\title{
COMPARATIVE STUDY BETWEEN SINGLE ANASTMOSIS DUODENO-ILEAL BYPASS-SLEEVE AND MINI-GASTRIC BYPASS AFTER FAILED SLEEVE GASTRECTOMY IN MORBIDLY OBESE PATIENTS
}

\author{
${ }^{1}$ Alaa Abbas Sabry Moustafa, ${ }^{1}$ Karim Sabry Abdel-Samee, ${ }^{2}$ Abdallah Hamed \\ Ibrahim, ${ }^{2}$ Moustafa Mahmoud Emad Mohamed
}

${ }^{1}$ Bariatric Surgery Unit and ${ }^{2}$ Department of General Surgery, Faculty of MedicineAin Shams University, Cairo Egypt.

Corresponding author: Moustafa Mahmoud Emad Mohamed

Mobile: 01000608975

E mail:

Moustafaemad@hotmail.com

Received: 1/5/2019

Accepted:30/5/2019

\begin{abstract}
Background: About $10-30 \%$ of the SG patients are associated with long-term failure, due either to inadequate weight loss or to renewed weight gain.

Objective: To compare weight loss as well as the metabolic effects of 2 types of bariatric surgery after failure of primary SG; Single Anastomosis Duodeno-ileal Bypass-Sleeve (SADI-S) and MiniGastric bypass (MGB), in the first year postoperative follow up.

Patients and Methods: This study is a prospective controlled study which included 30 patients underwent bariatric and metabolic surgeries at Ain-Shams University El-Demerdash Hospital, Cairo, Egypt from July 2017 to July 2018 with one year of postoperative follow up till July 2019. A comprehensive assessment program was carefully structured so that a disciplined routine is followed in each patient. All patients were preoperatively evaluated with provision of extensive information and consented to participate in the study.
\end{abstract}

Results: According to our results, the SADI-S group presented markedly higher percentage of EWL of $91.4 \%$ vs $71.6 \%$ in MGB after one year. Control of DM, with HbA1c below 6\%, was obtained in $86.6 \%$ in both groups with more decrease in mean HbA1c of SADIS being 5.44 vs $5.815 \%$ in MGB after one year. Most patients abandoned antidiabetic therapy or at least were controlled by less medications and lower doses. The SADI-S group presented remission of hypertension by $81.8 \%$ of patients vs $80 \%$ in MGB with far less medications. Lipid profile improvement was noticed in both groups with slightly higher resolution in SADIS group by $93.3 \%$ vs $86.6 \%$ for total cholesterol, $86.6 \%$ vs $80 \%$ for T.G, $80 \%$ vs $86.6 \%$ for LDL, $60 \%$ vs $67.6 \%$ for HDL in SADIS and MGB patients respectively. Although the nutritional deficiency is still a considerable concern after SADIS, ours study didn't show intense difference from MGB provided that proper vitamin supplementation and patient compliance are maintained postoperatively.

Conclusion: When compared to gastric bypass, SADI-S appears to be an effective and safe therapeutic technique as a revisional surgery for failed primary SG with excellent short-term results for treating morbid obesity and its associated comorbidities with a low rate of nutritional complications. Proving its safety and efficacy by further studies and long term follow up will grant it more popularity in the future.

Keywords: Single anastomosis duodeno-ileal bypass-sleeve, mini-gastric bypass. 


\section{INTRODUCTION}

Obesity is a pandemic health problem in both developed and developing countries and the costs of care continue to grow in parallel with the prevalence of the disease. This morbid condition leads to a high incidence of complications and a decrease in life expectancy, especially among younger adults $^{(1)}$.

Surgical treatment of morbid obesity results in significant sustained weight loss, which reduces obesity-related morbidity and increases survival compared with patients receiving optimal medical therapy ${ }^{(2)}$.

Laparoscopic sleeve gastrectomy (SG) has demonstrated its effectiveness in achieving weight loss and resolution of comorbidities in patients with severe obesity and super-obesity ${ }^{(3)}$.

However, this purely restrictive technique may sometimes be associated with long-term failure, due either to inadequate weight loss or to renewed weight gain; this requires effective low-morbidity alternative procedures $^{(4)}$.

Himpens et al. reported that more than $30 \%$ of the SG patients had inadequate weight loss or renewed weight gain, requiring an additional procedure ${ }^{(5)}$.

Patients with insufficient weight loss or weight regain can be treated surgically by a second intervention, such as the re- SG, Rebibo et al. ${ }^{(6)}$ the Roux-en-Y gastric bypass (RYGB) or the biliopancreatic diversion with duodenal switch ${ }^{(7)}$.

Laparoscopic mini-gastric bypass (LMGBP), described by Rutledge, has a lower operative morbidity with equivalent efficacy compared to laparoscopic Roux-en$\mathrm{Y}$ gastric bypass (RYGB), in terms of resolution of the metabolic syndrome, loss of excess weight (EWL) and improvement of the quality of life ${ }^{(8)}$.

Recently, the single-anastomosis duodeno-ileal bypass with sleeve gastrectomy (SADI-S) was introduced as a modified and simplified DS that has demonstrated satisfactory short-and long-term results. After this success, it was introduced as a second step after SG for insufficient weight loss ${ }^{(\boldsymbol{9})}$.

\section{AIM OF THE WORK}

The aim of this prospective study is to compare weight loss as well as the metabolic effects of 2 types of bariatric surgery after failure of primary SG; Single Anastomosis Duodeno-ileal Bypass-Sleeve (SADI-S) and Mini-Gastric bypass (MGB), in the first year postoperative follow up.

\section{PATIENTS AND METHODS}

This study is a prospective controlled study which included 30 patients underwent bariatric and metabolic surgeries at AinShams University El-Demerdash Hospital, Cairo, Egypt from July 2017 to July 2018 with one year of postoperative follow up till July 2019. Those patients were sub-divided into 2 groups; Group (A): Composed of 15 patients who underwent SADI- operation following failed primary SG. Group (B): Composed of 15 patients who underwent Mini-gastric bypass operation following failed primary SG.

A comprehensive assessment program was carefully structured so that a disciplined routine is followed in each patient. All patients were preoperatively evaluated with provision of extensive information and consented to participate in the study. 
Inclusion criteria: Inclusion criteria met the National institute of health criteria for bariatric surgery; Are fit for surgery. Adult male or female patients, age (18-60 years). Patients who have BMI $(35-\geq 40)$ 16 months following Sleeve gastrectomy or weight regain following primary Sleeve gastrectomy.

Exclusion criteria: Are generally unfit for operation. Old age patients more than 60 years or younger than 18 years. Patients with history of psychiatric illness. Patient refusal.

\section{Steps of study:}

\section{All patients were subjected to preoperative assessment which included:}

History taking: Personal history. History of obesity and weight loss trials. Detailed dietary history and eating habits. History of previous operations especially gastrointestinal surgery. Other systems review (cardio- vascular system, respiratory, liver diseases). Associated comorbidities e.g. endocrine disorder. Psychological status. Medications history.

\section{Examination:}

A- General: full general examination was done, focusing on: BMI and body circumferences measurement. Vital data. Complexion (pallor, jaundice). Cardiovascular fitness. Respiratory fitness.

B- Local: full abdominal examination focused on: Scars of previous operations (mainly in the upper abdomen). Abdominal wall hernias.

\section{Investigations:}

Laboratory: General pre-operative investigations for all the patients include: Complete blood picture. Coagulation profile. Liver function tests. Arterial blood gases.
Kidney function tests. Lipid profile. Thyroid profile. Haemoglobin A1C.

Patient was described as diabetic if fasting blood sugar was $126 \mathrm{mg} / \mathrm{dl}$ or above or two hours postprandial blood sugar was $200 \mathrm{mg} / \mathrm{dl}$ or above or random blood sugar was $200 \mathrm{mg} / \mathrm{dl}$ or above. Serum electrolytes including calcium. VitaminB12 and Vitamin D.

Cardio-respiratory investigations: All patients had ECG, Pulmonary function tests while some had echocardiography for cardiac troubles.

Radiological: All patients had preoperative chest X-ray and pelviabdominal ultrasound. CT volumtery and upper GI endoscopy if indicated. Preoperative co-morbid factors such as hypertension or electrolyte disturbance were corrected as much as possible.

All cases were operated by consultant surgeon and according to the standardized technique. Surgeries were done by the same surgical team throughout the study. As part of their preparation, SADI-S and MGB operations were described in details to the candidates for surgery and the surgical procedure was reviewed with them with the possibility of conversion to open surgery and all the possible intraoperative, early and late postoperative complications.

\section{Procedure:}

Preoperative medications: Two grams of third generation cephalosporin. Proton pump inhibitor. Anti-emetic.

\section{Surgical Technique}

\section{Steps of SADI-S operation:}

Surgery was performed under general anesthesia. The patients were placed in supine position with the table in reverse 
Trendelenburg position with legs spread. Nasogastric tube was inserted.

Creating Pneumoperitoneum \& Ports placement: Closed method using Veress needle at Palmer's point was used for establishing the pneumoperitoneum. In patients where the Veress cannot be inserted safely or has failed, optical entry using a zero-degree telescope is used for insertion of the first port. The open Hasson technique was also used in some cases. The intrabdominal pressure is maintained between 15 and $20 \mathrm{~mm}$. The optical $10 \mathrm{~mm}$ port is placed 2-3 finger breadths above the umbilicus in the left paramedian line. A 12 $\mathrm{mm}$ left-subcostal port is placed for the surgeon's right hand, and a $12 \mathrm{~mm}$ right paramedian port is used for the linear stapler and the surgeon's left hand. A $5 \mathrm{~mm}$ port in the left mid axillary line for the assistant. A $5 \mathrm{~mm}$ incision in the sub- xiphoid region for hook liver retractor to elevate the left lobe of liver.

Mobilization of the stomach: Dissection of adhesions between sleeved stomach and liver, resleeving of the stomach over $40 \mathrm{~F}$ bougie could be done using black and green cartridges.

The duodenal dissection: The dissection is commenced approximately $4-5$ $\mathrm{cm}$ distal to the pylorus by having the assistant grasp the antrum and retracting it laterally, which linearizes the first portion of duodenum. Using hook monopolar energy, the peritoneum overlying the inferior and superior portions of the duodenum is freed avoiding injury to the right gastric vessels and the supra-duodenal artery (preserving all the vascularization to the lesser curvature). The mobilization is performed until the point where the duodenum fuses posteriorly with the pancreas. The retro-duodenal dissection is performed bluntly with judicious bipolar cauterization of vessels creating a small window between the duodenum and pancreatic head wide enough to admit a linear stapler cartridge avoiding injury to the gastroduodenal artery as it passes behind the duodenal bulb.

Doudenal transection: The duodenum is divided $2-4 \mathrm{~cm}$ from the pylorus with a 60 mm blue linear stapler (EndoGIA).

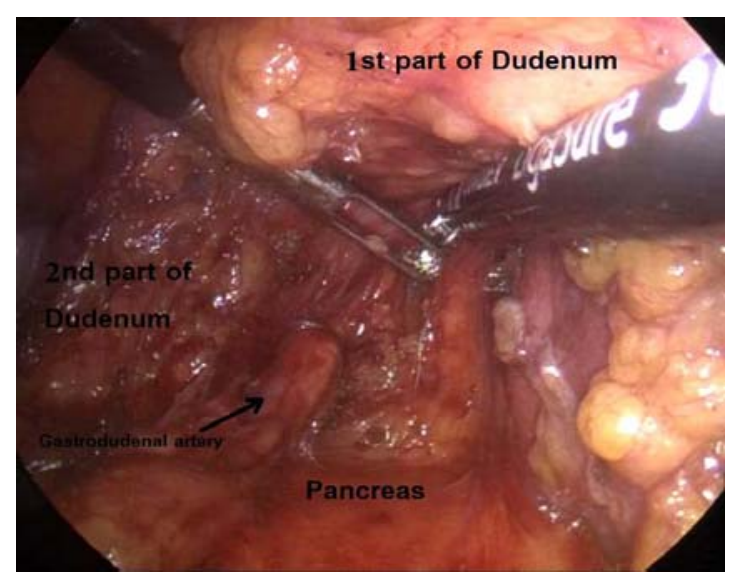

Figure (1): SADIS: Posterior blunt dissection between the duodenum and the pancreas creating a window for the stapler avoiding damage of gastroduodenal artery. 

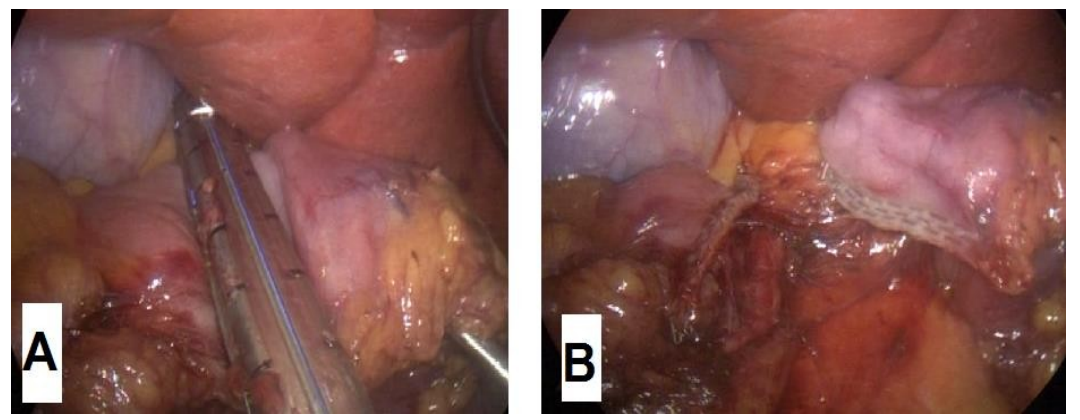

Figure (2): SADIS: Duodenal Transection A) Endo-GIA stapler is applied $3 \mathrm{~mm}$ from the pylorus. B) Duodenum divided.

Choosing \& measurement of ileal loop: colic fashion to the duodenal stump and an The ileo-cecal junction is identified. Twohundred and fifty centimeters are measured proximally along the ileum.

Doudeno-ileal anastomosis: The selected ioop is lifted cranially in an anteisoperistaltic end-to- side duodeno-ileal anastomosis is completed using either semimechanically with a $30-\mathrm{mm}$ linear stapler or hand sewn with sequential running sutures of a 3/0 PDS or Vicryl TM.

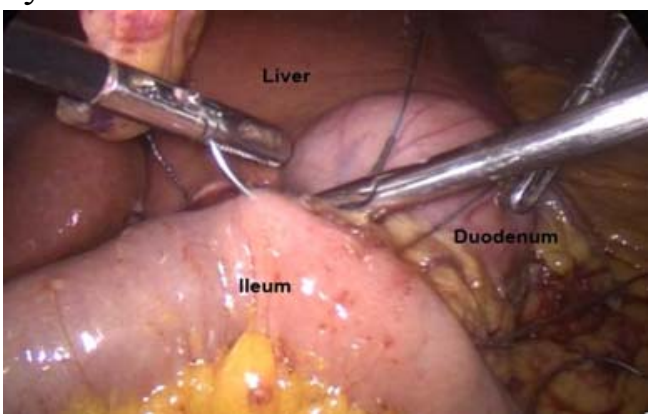

Figure (3): SADIS: Handswen end-to-side duodeno-ileal anastomosis.

Leak test: The anastomosis and staple instillation through the nasogastric tube and line are tested for leaks with methylene blue a suction drain is left behind.

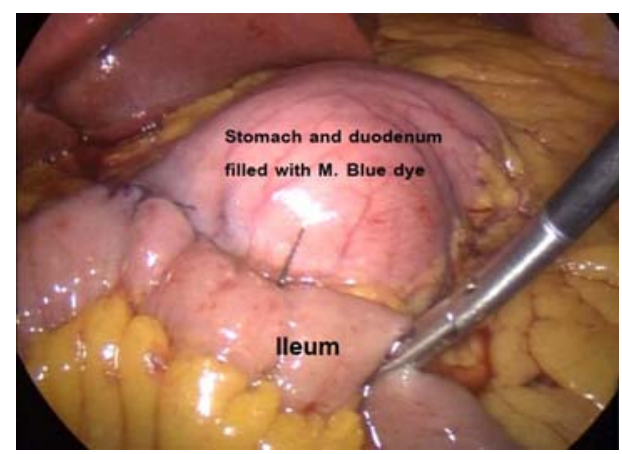

Figure (4): SADIS: Intact duodenoileal anastomosis after methylene blue dye inflation of the stomach and duodenum.

\section{Steps of MGB operation:}

Surgery was performed under general anesthesia. The patients were placed in supine position with the table in reverse
Trendelenburg position with legs spread. Nasogastric tube was inserted.

Creating Pneumoperitoneum \& Ports placement: After Veress needle insufflation 
in the left hypochondrium, the first 11-mm trocar for the camera was placed in the midpoint between the xiphoid and the umbilicus at mid line. The second trocar (5 $\mathrm{mm}$ ) was placed in the right hypochondrium at anterior axillary line; the third trocar (12 $\mathrm{mm}$ ) was inserted in the left hypochondrium, symmetrical to the previous one. The fourth trocar $(12 \mathrm{~mm})$ was placed in the right quadrant at anterior clavicular line on the same level of the camera. A $5 \mathrm{~mm}$ incision in the sub-xiphoid region for hook liver retractor to elevate the left lobe of liver.
Creation of the gastric pouch: After dissection of adhesions between sleeved stomach and liver, the stomach is divided at the junction of the body and the antrum at the level of the crow's foot with $45-\mathrm{mm}$ Endo- GIA stapler to get the longest possible gastric pouch. A lesser curvature-based tube of stomach is constructed with a $60-\mathrm{mm}$ linear stapler using black or green cartridges around an orogastric tube of $36 \mathrm{Fr}$ size.

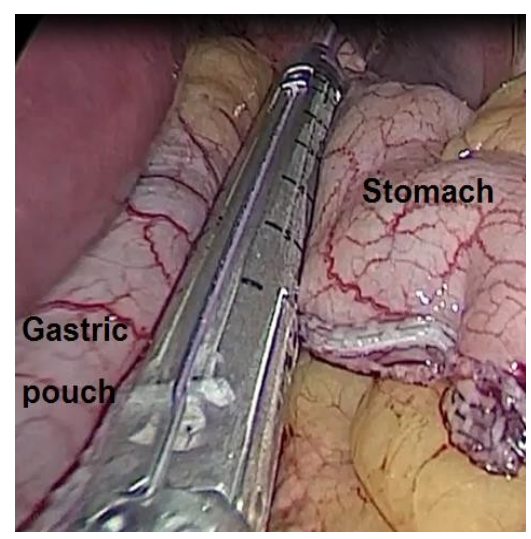

Figure (5): MGB: Creation of the gastric pouch along Lesser curvature of the stomach.

Choosing \& Measurement of jejunal loop: A graded grasper is used to measure about $200 \mathrm{~cm}$ of jejunum from the ligament of Treitz.

Creation of gastrojejunostomy: The jejunal loop brought up antecolic and anastomosed to the stomach tube with 45 - mm Endo-GIA stapler. Antireflux stitch between biliary limb and gastric pouch was made to decrease biliary reflux. The common stapling defect was closed over nasogastric tube with two layers of No 2-0 absorbable Vicryl TM suture in a running fashion.

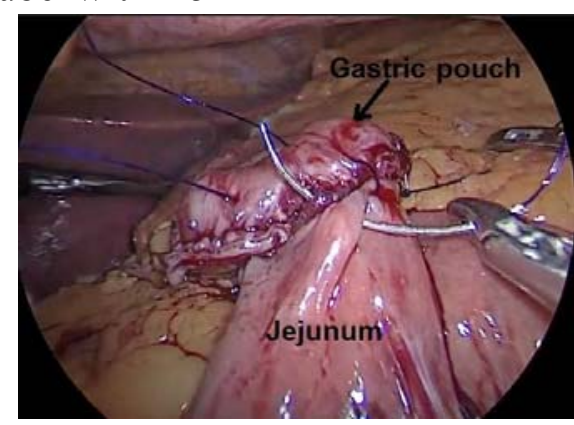

Figure (6): MGB: Closure of the stapling defect of the gastrojejunostomy.

Leak test: The anastomosis was then tested with methylene blue injected through the nasogastric tube. A tube drain was left in the vicinity of the gastrojejunostomy under the left lobe of the liver. 
All patients were given a standard regimen of multivitamins, including vitamin B12, iron supplementation and calcium. A 6month course of proton pump inhibitors (omeprazole $40 \mathrm{mg}$ ) was prescribed to all patients. A consistent exercise program was emphasized to all patients.

\section{Postoperative follow up:}

The follow up period of one year was carried out on an outpatient basis: Weekly visit for one month after discharge from the hospital. Monthly visit till the end of the third month. Visit every three months till the end of the first follow-up year. In each visit patient had: Full clinical assessment, Measurement of the anthropometric measures, Required investigations as indicated and according to study plan follow up.

\section{Data Management and Analysis:}

The collected data was revised, coded, tabulated and introduced to a PC using Statistical package for Social Science. Data was presented, and suitable analysis was done according to the type of data obtained for each parameter.

Outcome measures: The comparison between SADI-S and Mini-gastric bypass regarding the following endpoints after 6 months and one year; Weight loss. Metabolic outcome (D.M \& hypertension control and lipid profile). Nutritional deficiencies of Vitamin D, Vitamin B12, Calcium and albumin.

Descriptive statistics: Mean. Standard deviation $( \pm$ SD). Minimum and maximum values (range) for numerical data. Frequency and percentage of non-numerical data.

\section{Analytical statistics: Independent} Samples T: Test was used to assess the statistical significance of the difference between the two-study group means. Correlation analysis (using Pearson's method): To assess the strength of associateion between two quantitative variables. The correlation coefficient denoted symbolically " $r$ " defines the strength and direction of the linear relationship between two variables. Paired t-test: was used to assess the statistical significance of the difference between two means of one quantitative variable measured twice for the same study group. P- value (level of significance): $\mathbf{P}>$ 0.05: Non-significant $(\mathrm{NS}) . \quad \mathbf{P}<\mathbf{0 . 0 5}$ : Significant (S). $\mathbf{P}<$ 0.01: Highly significant (HS). Data were graphically represented using Power Point program.

\section{RESULTS}

This study included 30 failed sleeve gastrectomy patients divided into 2 groups; Group (A) of 15 patients who underwent SADI and group (B) of 15 patients who underwent MGB.

Group (A) SADI patients included 7 males (46.6\% of patients) and 8 females $(53.3 \%)$ with mean age of $49.0 \pm 6.2$ years while Group (B) MGB patients included 6 males $(40 \%)$ and 9 females $(60 \%)$ with mean age of $48.5 \pm 8.7$ years. Table (1)

Group (A) patients had mean preoperative body mass index (BMI) of 47.3 $\pm 4.1 \mathrm{Kg} / \mathrm{m} 2$, and the mean preoperative weight of $129.3 \pm 11.3 \mathrm{Kg}$. Group (B) had a mean preoperative body mass index (BMI) of $46 \pm 3.6 \mathrm{~kg} / \mathrm{m} 2$ and mean preoperative weight of $129.8 \pm 11.5 \mathrm{~kg}$. The mean excess weight was $61.1 \pm 11.1 \mathrm{~kg}$ and $59.3 \pm 10.4$ $\mathrm{kg}$ in group (A) and (B) respectively. Table (1) 
Table (1): Comparison between the SADIS and MGB group regarding preoperative demographic data.

\begin{tabular}{|c|c|c|c|c|}
\hline Demographic data & Group A (SADIS) & Group B (MGB) & \multirow{2}{*}{ Test } & \multirow{2}{*}{$\begin{array}{l}\text { P-value } \\
\text { (Sig.) }\end{array}$} \\
\hline Number of patients & No. $=15$ & No. $=15$ & & \\
\hline \multicolumn{5}{|l|}{ Age (years) } \\
\hline Mean \pm SD & $48.5 \pm 6.1$ & $48 \pm 8.6$ & \multirow[t]{2}{*}{0.184} & \multirow[t]{2}{*}{0.855} \\
\hline Median (Range) & $47(39-60)$ & $48(35-60)$ & & \\
\hline \multicolumn{5}{|l|}{ Gender } \\
\hline Female & $8(53.3 \%)$ & $9(60 \%)$ & \multirow[t]{2}{*}{0.136} & \multirow[t]{2}{*}{0.712} \\
\hline Male & $7(46.6 \%)$ & $6(40 \%)$ & & \\
\hline \multicolumn{5}{|l|}{ Weight (Kg) } \\
\hline Mean \pm SD & $129.3 \pm 11.3$ & $129.8 \pm 11.5$ & \multirow[t]{2}{*}{0.120} & \multirow[t]{2}{*}{0.905} \\
\hline Median (Range) & $128.5(107-149)$ & $129(111-152)$ & & \\
\hline \multicolumn{5}{|l|}{ Excess weight $(\mathrm{Kg})$} \\
\hline Mean \pm SD & $61.1 \pm 11.1$ & $59.3 \pm 10.4$ & \multirow[t]{2}{*}{0.458} & \multirow[t]{2}{*}{0.650} \\
\hline Median (Range) & $60(41-76)$ & $60.5(42.5-81.5)$ & & \\
\hline \multicolumn{5}{|l|}{ Height $(\mathrm{m})$} \\
\hline Mean \pm SD & $1.64 \pm 0.05$ & $1.67 \pm 0.04$ & \multirow[t]{2}{*}{1.815} & \multirow[t]{2}{*}{0.080} \\
\hline Median (Range) & $1.67(1.56-1.74)$ & $1.68(1.59-1.74)$ & & \\
\hline \multicolumn{5}{|l|}{$\operatorname{BMI}\left(\mathrm{Kg} / \mathrm{m}^{2}\right)$} \\
\hline Mean \pm SD & $47.3 \pm 4.1$ & $46 \pm 3.6$ & \multirow[t]{2}{*}{0.923} & \multirow[t]{2}{*}{0.364} \\
\hline Median (Range) & $46.75(38.6-53.9)$ & $47(39-53.9)$ & & \\
\hline
\end{tabular}

Co-morbidities of patients included diabetes $(100 \%)$ in both groups, with 7 patients $(46.6 \%)$ of group (A) on oral hypoglycaemics and 8 patients $(53.3 \%)$ on insulin therapy with mean preoperative $\mathrm{HbAlc}$ of $7.77 \pm 1.14 \%$. Meanwhile 6 patients $(40 \%)$ of group B were on oral hypoglycaemics and the other 9 patients $(60 \%)$ on insulin therapy with mean preoperative $\mathrm{HbA1c}$ of $7.66 \pm 0.5$ $\%$. Tables $(2,3)$.
Blood levels of Vit D3, calcium, VitB12, hemoglobin and albumin were assessed preoperatively to exclude any underlying abnormalities, and all were rectified to be within normal range in all patients of both groups. Table (3)

Hypertensive patients under treatment constituted 11 patients $(73.3 \%)$ of group A patients and 10 patients (66.6\%) of group B. Table (2).

Table (2): Comparison between the studied groups regarding pre-operative medical data.

\begin{tabular}{|c|c|c|c|c|}
\hline Pre-operative medical data & Group A (SADIS) & Group B (MGB) & \multirow{2}{*}{ Test } & \multirow{2}{*}{ P-value (Sig.) } \\
\hline Number of patients & $15(100 \%)$ & $15(100 \%)$ & & \\
\hline \multicolumn{5}{|l|}{ DM treatment (\%) } \\
\hline Oral & $7(46.6 \%)$ & $6(40 \%)$ & \multirow[t]{2}{*}{0.136} & \multirow[t]{2}{*}{0.712} \\
\hline Insulin & $8(53.3 \%)$ & $9(60 \%)$ & & \\
\hline \multicolumn{5}{|l|}{ HTN (\%) } \\
\hline Absent & $4(26.6 \%)$ & $5(33.3 \%)$ & \multirow[t]{2}{*}{0.159} & \multirow[t]{2}{*}{0.690} \\
\hline Diagnosed and under TTT & $11(73.3 \%)$ & $10(66.6 \%)$ & & \\
\hline
\end{tabular}


Comparative study between single anastmosis duodeno-ileal bypass-sleeve and mini-gastric bypass...

Table (3): Comparison between the studied groups regarding pre-operative laboratory data.

\begin{tabular}{|c|c|c|c|c|}
\hline Pre-operative laboratory data & Group A (SADIS) & Group B (MGB) & Test & $\begin{array}{c}\text { P-value } \\
\text { (Sig.) }\end{array}$ \\
\hline \multicolumn{5}{|l|}{ HbA1c (\%) } \\
\hline Mean \pm SD & $7.66 \pm 1.14$ & $7.55 \pm 0.5$ & \multirow[t]{2}{*}{0.342} & \multirow[t]{2}{*}{0.734} \\
\hline Median (Range) & $7.6(6.2-9.5)$ & $7.5(6.7-8.6)$ & & \\
\hline \multicolumn{5}{|l|}{ Total cholesterol (mg/dL) } \\
\hline Mean \pm SD & $202 \pm 49.3$ & $201 \pm 47.1$ & \multirow[t]{2}{*}{0.057} & \multirow[t]{2}{*}{0.955} \\
\hline Median (Range) & $188(139-284)$ & $191(135-290)$ & & \\
\hline \multicolumn{5}{|l|}{ Triglycerides (mg/dL) } \\
\hline Mean \pm SD & $211 \pm 58.3$ & $216.5 \pm 44.1$ & \multirow[t]{2}{*}{0.291} & \multirow[t]{2}{*}{0.773} \\
\hline Median (Range) & $233(127-294)$ & $226.5(125-295)$ & & \\
\hline \multicolumn{5}{|l|}{$\mathrm{LDL}(\mathrm{mg} / \mathrm{dL})$} \\
\hline Mean \pm SD & $116.2 \pm 38.1$ & $100.8 \pm 25.0$ & \multirow[t]{2}{*}{1.309} & \multirow[t]{2}{*}{0.201} \\
\hline Median (Range) & $99(64-169)$ & $94.5(65-155)$ & & \\
\hline \multicolumn{5}{|l|}{$\mathrm{HDL}(\mathrm{mg} / \mathrm{dL})$} \\
\hline Mean \pm SD & $45.4 \pm 9.9$ & $43.1 \pm 6.6$ & \multirow[t]{2}{*}{0.749} & \multirow[t]{2}{*}{0.460} \\
\hline Median (Range) & $43(29-66)$ & $43.5(33-54)$ & & \\
\hline \multicolumn{5}{|l|}{ Vitamin D (ng/dl) } \\
\hline Mean \pm SD & $34.1 \pm 8.4$ & $35.7 \pm 9.5$ & \multirow[t]{2}{*}{0.489} & \multirow[t]{2}{*}{0.628} \\
\hline Median (Range) & $35(22-48)$ & $35.5(15-50)$ & & \\
\hline \multicolumn{5}{|l|}{ Calcium (mg/dl) } \\
\hline Mean \pm SD & $9.26 \pm 0.58$ & $9.55 \pm 0.52$ & \multirow[t]{2}{*}{1.442} & \multirow[t]{2}{*}{0.160} \\
\hline Median (Range) & $9.3(8.5-10.2)$ & $9.55(8.5-10.2)$ & & \\
\hline \multicolumn{5}{|l|}{ Vitamin B12 (pg/dl) } \\
\hline Mean \pm SD & $410.7 \pm 154.7$ & $414.8 \pm 92.0$ & \multirow[t]{2}{*}{0.088} & \multirow[t]{2}{*}{0.930} \\
\hline Median (Range) & $384.5(196-410)$ & $409(196-573)$ & & \\
\hline \multicolumn{5}{|l|}{ Hemoglobin (gm/dL) } \\
\hline Mean \pm SD & $13.6 \pm 0.8$ & $13.3 \pm 0.8$ & \multirow[t]{2}{*}{1.027} & \multirow[t]{2}{*}{0.313} \\
\hline Median (Range) & $13.8(12.3-15)$ & $13.5(12.2-15)$ & & \\
\hline \multicolumn{5}{|l|}{ Albumin (mg/dL) } \\
\hline Mean \pm SD & $4.23 \pm 0.64$ & $4.27 \pm 0.59$ & \multirow[t]{2}{*}{0.178} & \multirow[t]{2}{*}{0.860} \\
\hline Median (Range) & $4.15(3.3-5.4)$ & $4.15(3.5-5.3)$ & & \\
\hline
\end{tabular}

After 6 postoperative months, group (A) EWL of $29.4 \pm 6.0 \mathrm{~kg}$ and $41.5 \pm 7.8 \mathrm{~kg}$ SADIS patients had mean EWL of $43.5 \pm$ after 6 months and one year respectively $9.4 \mathrm{~kg}$ and $54.8 \pm 10.1 \mathrm{~kg}$ after one year. Table (4).

While group (B) MGB patients had mean

Table (4): Comparison between the studied groups regarding excess weight loss by (KG) at 6 months and at 12 months post-operative.

\begin{tabular}{|c|c|c|c|c|}
\hline Excess weight loss (Kg) & Group A (SADIS) & Group B (MGB) & Test & $\begin{array}{c}\text { P-value } \\
\text { (Sig.) }\end{array}$ \\
\hline At 6 months & $43.5 \pm 9.4 \mathrm{~kg}$ & $29.4 \pm 6.0 \mathrm{~kg}$ & \multirow{2}{|c|}{4.897} & $<0.001$ \\
\hline Mean \pm SD & $42.5(30-59) \mathrm{kg}$ & $29.25(21-43.5) \mathrm{kg}$ & \\
\hline Median Range) & $54.8 \pm 10.1 \mathrm{~kg}$ & $41.5 \pm 7.8 \mathrm{~kg}$ & \multirow{2}{*}{4.036} & 0.000 \\
\hline At 12 months & $54.25(37.5-70) \mathrm{kg}$ & $42.25(28.5-58) \mathrm{kg}$ & & \\
\hline Mean \pm SD &
\end{tabular}

For Group A patients mean percentage of EWL was $71.3 \pm 6.2 \%$ after 6 months and markedly increased to $90.7 \pm 3.0 \%$ after one year. However, in group B mean percentage of EWL was $50.1 \pm 2.3 \%$ and increased to $70.6 \pm 2.0 \%$ after one year. Table (5). 
Table (5): Comparison between the studied groups regarding percentage of excess weight loss at 6 months and at 12 months post-operative.

\begin{tabular}{|l|c|c|c|c|}
\hline Excess weight loss (\%) & Group A (SADIS) & Group B (MGB) & Test & $\begin{array}{c}\text { P-value } \\
\text { (Sig.) }\end{array}$ \\
\hline At 6 months & $71.3 \pm 6.2 \%$ & $50.1 \pm 2.3 \%$ & 12.416 & $<0.001$ \\
\hline Mean \pm SD & $73(49.2-78.9) \%$ & $50.7(46.2-54.6) \%$ & \\
\hline Median (Range) & $90.7 \pm 3.0 \%$ & $70.6 \pm 2.0 \%$ & 21.591 & $<0.001$ \\
\hline At 12 months & $91(82.5-95.5) \%$ & $70.85(68.3-76.2) \%$ & & \\
\hline Mean \pm SD & Median (Range) &
\end{tabular}

After 6 months of group A patients follow patients (40\%) were in need for treatment with

up, 4 patients $(26.6 \%)$ of patients still had resolution of 9 patients $(60 \%)$ of cases in the diagnosis of D.M with resolution occurred in first 6 months and increased to 13 patients 11 patients $(73.3 \%)$ which increased after one $\quad(86.6 \%)$ after one year leaving only 2 patients year to 13 patients $(86.6 \%)$ and only 2 patients $(13.3 \%)$ of patients still diabetic. In group B, 6 (13.3\%) diabetic Table (6).

Table (6): Comparison between the studied groups regarding the percentage of DM patient's preoperative, at 6 months and at 12 months post-operative.

\begin{tabular}{|l|c|c|c|c|}
\hline \multicolumn{1}{|c|}{ DM patients (\%) } & Group A (SADIS) & Group B (MGB) & \multirow{2}{*}{ Test } & \multirow{2}{*}{ P-value (Sig.) } \\
\hline Number of M.O Patients & $15(100 \%)$ & $15(100 \%)$ & & \multirow{2}{*}{0.000} \\
\hline Pre-operative & $15(100 \%)$ & $15(100 \%)$ & 0.000 & 0.438 \\
\hline At 6 months & $4(26.6 \%)$ & $6(40 \%)$ & 0.600 & 1.000 \\
\hline At 12 months & $2(13.3 \%)$ & $2(13.3 \%)$ & 0.000 & \\
\cline { 1 - 3 } Test & 26.25 & 23.656 & & \\
\cline { 1 - 3 } P-value (Sig.) & 0.000 & 0.000 & & \\
\hline
\end{tabular}

Regarding antidiabetic treatment, perMeanwhile, those in group B on oral centage of group A diabetic patients on oral treatment decreased from $46.6 \%$ (7 patients) to $0 \%$ (no patients) and those on insulin therapy decreased from $53.3 \%$ (8 patients) to $13.3 \% \quad(2$ patients $)$ after one year. treatment decreased from $40 \%$ (6 patients) to $6.6 \%$ (1 patient) and those on insulin decreased from $60 \%$ ( 9 patients) to $6.6 \%$ (1 patient) after one year Table (7).

Table (7): Comparison between the studied groups regarding the DM treatment pre-operative, at 6 months and at 12 months post-operative.

\begin{tabular}{|c|c|c|c|c|}
\hline DM treatment & Group A (SADIS) & Group B (MGB) & \multirow{2}{*}{ Test } & \multirow{2}{*}{ P-value (Sig.) } \\
\hline Number of Patients & $15(100 \%)$ & $15(100 \%)$ & & \\
\hline \multicolumn{5}{|l|}{ Pre-operative } \\
\hline No need for TTT & $0(0 \%)$ & $0(0 \%)$ & \multirow[t]{3}{*}{0.136} & \multirow[t]{3}{*}{0.934} \\
\hline Oral & $7(46.6 \%)$ & $6(40 \%)$ & & \\
\hline Insulin & $8(53.3 \%)$ & $9(60 \%)$ & & \\
\hline \multicolumn{5}{|l|}{ At 6 months } \\
\hline No need for TTT & $11(73.3 \%)$ & $9(60 \%)$ & \multirow[t]{3}{*}{0.676} & \multirow[t]{3}{*}{0.713} \\
\hline Oral & $1(6.6 \%)$ & $2(13.3 \%)$ & & \\
\hline Insulin & $3(20 \%)$ & $4(26.6 \%)$ & & \\
\hline \multicolumn{5}{|l|}{ At 12 months } \\
\hline No need for TTT & $13(86.6 \%)$ & $13(86.6 \%)$ & \multirow[t]{3}{*}{1.333} & \multirow[t]{3}{*}{0.513} \\
\hline Oral & $0(0 \%)$ & $1(6.6 \%)$ & & \\
\hline Insulin & $2(13.3 \%)$ & $1(6.6 \%)$ & & \\
\hline Test & 27.769 & 23.758 & & \\
\hline P-value (Sig.) & 0.000 & 0.000 & & \\
\hline
\end{tabular}


Following up mean $\mathrm{HbA1c}$ in group A showed decreased mean level to $6.12 \pm 0.8$ after 6 months and more decrease to $5.43 \pm$ 0.47 after one year. For group B the mean
HbAlc decreased to $6.36 \pm 0.92$ then to 5.80 \pm 0.68 after 6 months and one year respectively Table (8).

Table (8): Comparison between the studied groups regarding the HbA1c level pre-operative, at 6 months and at 12 months post-operative.

\begin{tabular}{|c|c|c|c|c|}
\hline HbA1c $(\%)$ & Group A (SADIS) & Group B (MGB) & Test & P-value (Sig.) \\
\hline \multicolumn{5}{|l|}{ Pre-operative } \\
\hline Mean \pm SD & $7.66 \pm 1.14$ & $7.55 \pm 0.5$ & \multirow[t]{2}{*}{0.342} & \multirow[t]{2}{*}{0.734} \\
\hline Median (Range) & $7.6(6.2-9.5)$ & $7.5(6.7-8.6)$ & & \\
\hline \multicolumn{5}{|l|}{ At 6 months } \\
\hline Mean \pm SD & $6.12 \pm 0.8$ & $6.36 \pm 0.92$ & \multirow{2}{*}{0.762} & \multirow[t]{2}{*}{0.452} \\
\hline Median (Range) & $5.8(5.2-7.9)$ & $5.85(5.3-7.8)$ & & \\
\hline \multicolumn{5}{|l|}{ At 12 months } \\
\hline Mean \pm SD & $5.43 \pm 0.47$ & $5.80 \pm 0.68$ & \multirow[t]{2}{*}{1.734} & \multirow[t]{2}{*}{0.094} \\
\hline Median (Range) & $5.3(4.7-6.3)$ & $5.65(5.1-7.5)$ & & \\
\hline Test & 12.324 & 9.364 & & \\
\hline P-value (Sig.) & $<0.001$ & $<0.001$ & & \\
\hline
\end{tabular}

Number of hypertensive patients on treatment decreased from $73.3 \% \quad(11$ patients) to $20 \%$ (3 patients) after 6 months to $13.3 \%$ (2 patients) after one year in Group
$66.6 \%$ (10 patients) preoperatively to $26.6 \%$ (4 patients) after 6 months with more decrease to $13.3 \%$ (2 patients) after one year Table (9).

A. Meanwhile in Group B it decreased from

Table (9): Comparison between the studied groups regarding the percentage of HTN patients preoperative, at 6 months and at 12 months post-operative.

\begin{tabular}{|l|c|c|c|c|}
\hline \multicolumn{1}{|c|}{ HTN patients (\%) } & Group A (SADIS) & Group B (MGB) & \multirow{2}{*}{ Test } & $\begin{array}{c}\text { P-value } \\
\text { (Sig.) }\end{array}$ \\
\hline Pre-operative & $15(100 \%)$ & $15(100 \%)$ & 0.159 & 0.690 \\
\hline At 6 months & $11(73.3 \%)$ & $10(66.6 \%)$ & 0.186 & 0.666 \\
\hline At 12 months & $3(20 \%)$ & $4(26.6 \%)$ & 0.000 & 1.000 \\
\hline Test & $2(13.3 \%)$ & $2(13.3 \%)$ & & \\
\cline { 1 - 3 } P-value (Sig.) & 14.159 & 10.086 & & \\
\cline { 1 - 3 }
\end{tabular}

Marked improvement of the lipid profile was reported in both groups with decrease in the mean blood levels of various components of lipid profile and decrease in number of patients with dyslipidaemia as shown in Tables $(10,11)$. 
Table (10): Comparison between the studied groups regarding the blood level of different component of lipid profile pre-operative and during follow up.

\begin{tabular}{|c|c|c|c|c|}
\hline Lipid profile & $\begin{array}{c}\text { Group A (SADIS) } \\
\text { Mean } \pm \text { SD }\end{array}$ & $\begin{array}{c}\text { Group B (MGB) } \\
\text { Mean } \pm \text { SD }\end{array}$ & Test & P-value (Sig.) \\
\hline \multicolumn{5}{|l|}{ Total cholesterol $(\mathrm{mg} / \mathrm{dL})$} \\
\hline Pre-operative & $202 \pm 49.3$ & $201 \pm 47.1$ & 0.056 & 0.955 \\
\hline 6 months & $169.2 \pm 35.7$ & $184.5 \pm 30.8$ & 1.257 & 0.219 \\
\hline 12 months & $142.5 \pm 31.1$ & $152.9 \pm 20.3$ & 1.085 & 0.287 \\
\hline Test & 8.554 & 7.511 & & \\
\hline P-value (Sig.) & 0.001 & 0.002 & & \\
\hline \multicolumn{5}{|l|}{ Triglycerides $(\mathrm{mg} / \mathrm{dL})$} \\
\hline Pre-operative & $211 \pm 58.3$ & $216.5 \pm 44.1$ & 0.291 & 0.773 \\
\hline 6 months & $159.7 \pm 54.4$ & $182.4 \pm 39.2$ & 1.311 & 0.201 \\
\hline 12 months & $117.6 \pm 42.5$ & $152.8 \pm 33.8$ & 2.511 & 0.018 \\
\hline Test & 12.059 & 9.889 & & \\
\hline P-value (Sig.) & 0.000 & 0.000 & & \\
\hline \multicolumn{5}{|l|}{$\mathrm{LDL}(\mathrm{mg} / \mathrm{dL})$} \\
\hline Pre-operative & $116.2 \pm 38.1$ & $100.8 \pm 25.0$ & 1.309 & 0.201 \\
\hline 6 months & $93.8 \pm 24.4$ & $90.5 \pm 18.1$ & 0.421 & 0.677 \\
\hline 12 months & $78.2 \pm 19.9$ & $86.6 \pm 18.7$ & 1.191 & 0.243 \\
\hline Test & 6.721 & 5.860 & & \\
\hline P-value (Sig.) & 0.003 & 0.018 & & \\
\hline \multicolumn{5}{|l|}{$\mathrm{HDL}(\mathrm{mg} / \mathrm{dL})$} \\
\hline Pre-operative & $45.4 \pm 9.9$ & $43.1 \pm 6.6$ & 0.749 & 0.460 \\
\hline 6 months & $46.9 \pm 10$ & $44.7 \pm 8.9$ & 0.636 & 0.529 \\
\hline 12 months & $47.3 \pm 9.1$ & $45.6 \pm 8.4$ & 0.532 & 0.599 \\
\hline Test & 0.161 & 0.373 & & \\
\hline P-value (Sig.) & 0.852 & 0.691 & & \\
\hline
\end{tabular}

Table (11): Comparison between the studied groups regarding the number of patients with abnormal lipid profile pre-operative and during follow up. (with $\%$ of diseased patients).

\begin{tabular}{|c|c|c|c|c|}
\hline \multirow{2}{*}{$\begin{array}{c}\% \text { Patients with abnormal lipid profile } \\
\text { Number of M.O Patients }\end{array}$} & Group (SADIS) & Group B (MGB) & \multirow[t]{2}{*}{ Test } & \multirow{2}{*}{$\begin{array}{l}\text { P-value } \\
\text { (Sig.) }\end{array}$} \\
\hline & $15(100 \%)$ & $15(100 \%)$ & & \\
\hline \multicolumn{5}{|l|}{ Total cholesterol } \\
\hline Pre-operative & $5(33.3 \%)$ & $6(40 \%)$ & 0.144 & 0.704 \\
\hline 6 months & $2(13.3 \%)$ & $3(20 \%)$ & 0.240 & 0.624 \\
\hline 12 months & $1(6.6 \%)$ & $2(13.3 \%)$ & 0.370 & 0.543 \\
\hline Test & 3.953 & 3.128 & & \\
\hline P-value (Sig.) & 0.138 & 0.209 & & \\
\hline \multicolumn{5}{|l|}{ Triglycerides } \\
\hline Pre-operative & $10(66.6 \%)$ & $9(60 \%)$ & 0.144 & 0.704 \\
\hline 6 months & $5(33.3 \%)$ & $6(40 \%)$ & 0.144 & 0.704 \\
\hline 12 months & $2(13.3 \%)$ & $3(20 \%)$ & 0.240 & 0.624 \\
\hline Test & 9.265 & 5.000 & & \\
\hline P-value (Sig.) & 0.010 & 0.082 & & \\
\hline \multicolumn{5}{|l|}{ LDL } \\
\hline Pre-operative & $7(46.6 \%)$ & $5(33.3 \%)$ & 0.556 & 0.455 \\
\hline 6 months & $5(33.3 \%)$ & $4(26.6 \%)$ & 0.159 & 0.690 \\
\hline 12 months & $3(20 \%)$ & $2(13.3 \%)$ & 0.322 & 0.570 \\
\hline Test & 2.400 & 1.684 & & \\
\hline P-value (Sig.) & 0.301 & 0.431 & & \\
\hline \multicolumn{5}{|l|}{ HDL } \\
\hline Pre-operative & $5(33.3 \%)$ & $6(40 \%)$ & 0.144 & 0.704 \\
\hline 6 months & $6(40 \%)$ & $6(40 \%)$ & 0.000 & 1.000 \\
\hline 12 months & $6(40 \%)$ & $5(33.3 \%)$ & 0.144 & 0.704 \\
\hline Test & 0.189 & 0.189 & & \\
\hline P-value (Sig.) & 0.910 & 0.910 & & \\
\hline
\end{tabular}


Comparing Vit D3 levels in both study groups showed decrease in its mean level from $34.1 \pm 8.4 \mathrm{ng} / \mathrm{mL}$ to $20.2 \pm 6.6 \mathrm{ng} / \mathrm{mL}$ in group A. While in group B it decreased from $35.7 \pm 9.5 \mathrm{ng} / \mathrm{mL}$ to $21.8 \pm 8.6 \mathrm{ng} / \mathrm{mL}$

Table (12): Comparison between the studied groups regarding the laboratory data of different nutrients pre- operatively and during follow up.

\begin{tabular}{|c|c|c|c|c|}
\hline Laboratory data & $\begin{array}{c}\text { Group A (SADIS) } \\
\text { Mean } \pm \text { SD }\end{array}$ & $\begin{array}{c}\text { Group B (MGB) } \\
\text { Mean } \pm \text { SD }\end{array}$ & Test & $\begin{array}{l}\text { P-value } \\
\text { (Sig.) }\end{array}$ \\
\hline \multicolumn{5}{|l|}{ Vitamin D (ng/ml) } \\
\hline Pre-operative & $34.1 \pm 8.4$ & $35.7 \pm 9.5$ & 0.489 & 0.628 \\
\hline 6 months & $21.2 \pm 11.6$ & $20.8 \pm 9.9$ & 0.102 & 0.919 \\
\hline 12 months & $20.2 \pm 6.6$ & $21.8 \pm 8.6$ & 0.572 & 0.572 \\
\hline Test & 10.876 & 11.905 & & \\
\hline P-value (Sig.) & 0.000 & 0.000 & & \\
\hline \multicolumn{5}{|l|}{ Calcium (mg/dl) } \\
\hline Pre-operative & $9.26 \pm 0.58$ & $9.55 \pm 0.52$ & 1.442 & 0.160 \\
\hline 6 months & $9.11 \pm 0.58$ & $9.21 \pm 0.38$ & 0.559 & 0.581 \\
\hline 12 months & $8.52 \pm 0.15$ & $8.76 \pm 0.33$ & 2.564 & 0.016 \\
\hline Test & 9.904 & 13.493 & & \\
\hline P-value (Sig.) & 0.000 & 0.000 & & \\
\hline \multicolumn{5}{|l|}{ Vitamin B12 (pg/ml) } \\
\hline Pre-operative & $410.7 \pm 154.7$ & $414.8 \pm 92.0$ & 0.088 & 0.930 \\
\hline 6 months & $363.5 \pm 139.8$ & $372.4 \pm 86.7$ & 0.210 & 0.835 \\
\hline 12 months & $228.4 \pm 28.3$ & $268.2 \pm 69.1$ & 2.064 & 0.048 \\
\hline Test & 9.098 & 20.834 & & \\
\hline P-value (Sig.) & 0.001 & 0.000 & & \\
\hline \multicolumn{5}{|l|}{$\begin{array}{l}\text { Hemoglobin } \\
(\mathrm{gm} / \mathrm{dL})\end{array}$} \\
\hline Pre-operative & $13.6 \pm 0.8$ & $13.3 \pm 0.8$ & 1.027 & 0.313 \\
\hline 6 months & $13.1 \pm 1.3$ & $12.6 \pm 0.8$ & 1.269 & 0.215 \\
\hline 12 months & $12.7 \pm 1.2$ & $12.5 \pm 0.8$ & 0.537 & 0.596 \\
\hline Test & 2.427 & 4.453 & & \\
\hline P-value (Sig.) & 0.101 & 0.018 & & \\
\hline \multicolumn{5}{|l|}{ Albumin $(\mathrm{mg} / \mathrm{dL})$} \\
\hline Pre-operative & $4.23 \pm 0.64$ & $4.27 \pm 0.59$ & 0.178 & 0.860 \\
\hline 6 months & $3.77 \pm 0.23$ & $3.92 \pm 0.41$ & 1.236 & 0.226 \\
\hline 12 months & $3.75 \pm 0.12$ & $3.81 \pm 0.24$ & 0.866 & 0.393 \\
\hline Test & 6.957 & 4.525 & & \\
\hline P-value (Sig.) & 0.002 & 0.017 & & \\
\hline
\end{tabular}

after one year. Meanwhile Ca levels dropped as well in both groups into $8.52 \pm 0.15 \mathrm{mg} / \mathrm{dl}$ and $8.76 \pm 0.33 \mathrm{mg} / \mathrm{dl}$ after one year in group A and B respectively Table (12). 
Table (13): Comparison between the studied groups regarding patients with abnormal laboratory data of different nutrients pre-operatively and during follow up.

\begin{tabular}{|c|c|c|c|c|}
\hline $\begin{array}{c}\% \text { Patients with abnormal } \\
\text { laboratory data }\end{array}$ & $\begin{array}{l}\text { Group A } \\
\text { (SADIS) }\end{array}$ & $\begin{array}{l}\text { Group B } \\
\text { (MGB) }\end{array}$ & \multirow[t]{2}{*}{ Test } & \multirow[t]{2}{*}{ P-value (Sig.) } \\
\hline Number of M.O patients & $15(100 \%)$ & $15(100 \%)$ & & \\
\hline \multicolumn{5}{|l|}{ Vitamin D } \\
\hline Pre-operative & $0(0 \%)$ & $0(0 \%)$ & 0.000 & 1.000 \\
\hline 6 months & $11(73.3 \%)$ & $9(60 \%)$ & 0.600 & 0.438 \\
\hline 12 months & $8(53.3 \%)$ & $6(40 \%)$ & 0.536 & 0.464 \\
\hline Test & 17.672 & 12.600 & & \\
\hline P-value (Sig.) & 0.000 & 0.002 & & \\
\hline \multicolumn{5}{|l|}{ Calcium } \\
\hline Pre-operative & $0(0 \%)$ & $0(0 \%)$ & 0.000 & 1.000 \\
\hline 6 months & $1(6.6 \%)$ & $1(6.6 \%)$ & 0.000 & 1.000 \\
\hline 12 months & $2(13.3 \%)$ & $1(6.6 \%)$ & 0.370 & 0.543 \\
\hline Test & 2.143 & 1.047 & & \\
\hline P-value (Sig.) & 0.342 & 0.592 & & \\
\hline \multicolumn{5}{|l|}{ Vitamin B12 } \\
\hline Pre-operative & $0(0 \%)$ & $0(0 \%)$ & 0.000 & 1.000 \\
\hline 6 months & $3(20 \%)$ & $1(6.6 \%)$ & 1.154 & 0.282 \\
\hline 12 months & $2(13.3 \%)$ & $1(6.6 \%)$ & 0.370 & 0.543 \\
\hline Test & 3.150 & 1.047 & & \\
\hline P-value (Sig.) & 0.207 & 0.592 & & \\
\hline \multicolumn{5}{|l|}{ Hemoglobin $(\mathrm{gm} / \mathrm{dL})$} \\
\hline Pre-operative & $0(0 \%)$ & $0(0 \%)$ & 0.000 & 1.000 \\
\hline 6 months & $2(13.3 \%)$ & $1(6.6 \%)$ & 0.370 & 0.543 \\
\hline 12 months & $3(20 \%)$ & $2(13.3 \%)$ & 0.240 & 0.624 \\
\hline Test & 3.150 & 2.143 & & \\
\hline P-value (Sig.) & 0.207 & 0.342 & & \\
\hline \multicolumn{5}{|l|}{ Albumin (mg/dL) } \\
\hline Pre-operative & $0(0 \%)$ & $0(0 \%)$ & 0.000 & 1.000 \\
\hline 6 months & $0(0 \%)$ & $0(0 \%)$ & 0.000 & 1.000 \\
\hline 12 months & $0(0 \%)$ & $0(0 \%)$ & 0.000 & 1.000 \\
\hline Test & 0.000 & 0.000 & & \\
\hline P-value (Sig.) & 1.000 & 1.000 & & \\
\hline
\end{tabular}

\section{DISCUSSION}

SADI-S operation was introduced to treat morbid obesity and its metabolic complications Sánchez-Pernaute ${ }^{(10)}$. The modification was devised to simplify previous successful operation of duodenal switch by Scopinaro et al. ${ }^{(11)}$, keeping the malabsorptive principles but attempting to decrease the operative complexity and, thus, the rate of surgical complications.

An adequate initial weight loss was predicted, because the restrictive component of the operation was a sleeve gastrectomy and the intestinal bypass was longer than that of the mini bypass Rutledge $e^{(12)}$. Thus, in terms of weight loss and co-morbidities resolution, SADI-S should initially provide satisfactory results.

We herein present the results of a prospective study started 2 years ago, our patients who had SADIS operation (group A) and MGB operation (group B) following failed primary SG were followed up for one year after surgery, examined and investigated for the outcome of surgery. Weight loss results, metabolic outcome and the nutritional effects were discussed as well as its way of management. 
Our study included 30 patients in the period from July 2017 to July 2018. Selection criteria were: Are fit for surgery. Adult male or female patients, age (18-60 years). Patients who have BMI (35 - $\geq 40$ ) 16 months following Sleeve gastrectomy or weight regain following primary Sleeve gastrectomy

A study done by Carbajo et al. (13) showed the mean EWL after 1 year of MGB is $75 \%$. Rutledge and Walsh ${ }^{(14)}$ reported the mean EWL after 1 year of MGB to be $80 \%$. Another study done by Noun et al. (15) proved the mean EWL after 1 year of MGB to be $69.9 \%$.

A study done by Sánchez-Pernaute et al. ${ }^{(16)}$ showed the mean EWL after 1 year of SADIS was $94 \%$ and another study by Sánchez-Pernaute et al. ${ }^{(9)}$ showed the mean EWL after 1 year of SADIS was 95\%. Moreover, Sánchez-Pernaute et al. ${ }^{(17)}$ showed the mean EWL in patients underwent SADIS was $73 \%$ at 6 months and $91 \%$ after one year.

Postoperative antidiabetic treatment was maintained upon decision of the endocrinologist, based on current ADA criteria.

Our study found that resolution of DM in SADIS patients initially occurred in $73.3 \%$ after 6 months increased to $86.6 \%$ after one year. However, in MGB patients it was $46.6 \%$ after 6 months and reached $86.6 \%$ after one year. Remission rates of SADIS patients under oral therapy is about $100 \%$ while MGB patients it was $93.3 \%$, whereas patients under insulin therapy the number markedly goes down with shift to oral therapy or marked decrease in insulin requirements to control D.M.

A study done be Del Gid et al. ${ }^{(18)}$ showed resolution of DM in patients underwent SADIS was $85 \%$ of cases versus $67 \%$ in those who had MGB after one year. Another study done be Sánchez-Pernaute et al. ${ }^{(17)}$ showed resolution of DM in patients underwent SADIS was $82 \%$ of cases after one year.

A study done by Rutledge and Walsh ${ }^{(14)}$ DM resolution 83\%. Meanwhile, Noun et al. $^{(15)}$ reported DM resolution in $85 \%$ of cases one year after MGB operation.

As Schauerre et al. (19) marks it is difficult to compare different studies because there is a huge variation in the severity of the populations included, and different criteria are used to define improvement or remission ${ }^{(20)}$.

In the present study we base on glycated hemoglobin and on the need for medical treatment, to define remission and control of the disease; HbAlc is the best way to determine diabetes control ${ }^{(21)}$ and it was recently found that a simpler approach to evaluate remission, based on $\mathrm{HbAlc}$ and the absence of medication, has the same value as more complex criteria ${ }^{(22)}$. We, along with others, consider remission with $\mathrm{HbAlc}$ values below $6 \%{ }^{(23)}$, which is the currently the most frequently used level.

Although DM resolution in our study appears to be the same ( $86.6 \%$ of patients) in both groups after one year, it is quite evident that the mean $\mathrm{HbA1c}$ level after one year in SADIS patient is less than that of MGB patients being $5.44 \pm 0.47 \%$ in SADIS group and $5.81 \pm 0.68 \%$ in MGB group which indicates more intense metabolic effect of SADIS to some extent in terms of DM control.

A study done by Sánchez-Pernaute et $a .^{(9)}$ on SADIS patients showed that hypertension was controlled in $98 \%$ of the patients, with absolute remission in $58 \%$ of the patients. A study done by Sánchez- 


\section{Alaa Abbas Sabry Moustafa, et al.,}

Pernaute et al. ${ }^{(17)}$ showed that SADIS patients had hypertension remitted in $52 \%$ of the patients and improved in $44 \%$.

One year follow up after MGB a study done by Rutledge and Walsh (14) showed hypertension resolution in $80 \%$ of cases. Whereas, a study done by Wang et al. ${ }^{(24)}$ showed hypertension resolution 94\% and another study done by Noun et al. ${ }^{(15)}$ reported hypertension resolution in $85 \%$.

As reported by our study, remission of hypertension included both absolute remission and improved control of the disease with less medication or by diet control alone without medication. It was difficult to ascertain complete resolution of hypertension in specific patients due to the poor compliance of some patients to diet control and medication, however it is statistically proved that there is $86.6 \%$ improved control rate in both groups.

Although, SADIS group patients were less dependent on medication than MGB group, the disease control was achieved by few antihypertensive medications in both groups as compared to preoperative medications in each group individually.

A study done by Sánchez-Pernaute et al. ${ }^{(9)}$ on SADIS patients showed $96 \%$ of the patients had normal total cholesterol levels, $73 \%$ of the patients had normal triglycerides level, 95\% had normal values of LDL and $73 \%$ had normal levels of HDL after one year.

A study done by Sánchez-Pernaute et al. ${ }^{(17)}$ showed Lipid profile improved in SADIS patients after one post- operative year, 96\% normal total cholesterol values, $89 \%$ of the patients had normal triglycerides, $79 \%$ normal LDL and $65 \%$ had normal HDL.
Rutledge $^{(12)}$ study showed resolution of elevated cholesterol in $93 \%$ and resolution of elevated triglycerides in $100 \%$ after one year of MGB. Whereas another study by Rutledge and Walsh ${ }^{(14)}$ reported resolution of hypercholesterolaemia in $89 \%$ of patients.

Most of studies have indicated achieving over $85 \%$ of dyslipidaemia resolution in one year generally with limited studies specifying percentages or levels for each category of the lipid profile individually.

From our results, it appears that SADIS operation appears to be slightly superior to MGB in terms of dyslipidaemia resolution generally and individually for each category of the lipid profile with much decrease in the mean levels as compared to those of MGB operation.

A study done by Sánchez-Pernaute et al. ${ }^{(17)}$ showed abnormal levels of Vit D in $50 \%$ of SADIS patients after one year. Another study done by Sánchez-Pernaute et al. $^{(9)}$ on patients one year after SADIS showed $0 \%$ of patients with hypocalcaemia and $46 \%$ of patients with abnormal blood levels of Vit. D.

Slater et al. (25) found vitamin D deficiencies in $57 \%$ of patients at one-year follow up after MGB. Brolin et al. ${ }^{(26)}$ found that $51 \%$ of postoperative patients had significant vitamin D deficiencies at two-year follow up.

Although various studies report different incidence of vitamin D deficiency and manifest calcium deficiency, this can be attributed not only to the proper vitamins supplementation, doses and GIT absorption, but also it is dependent mainly on patient compliance and commitment to vitamin supplementation after surgery. 
Vit D deficiency is described nowadays in most of literature as two groups, those with deficiency where the level in 10-20 $\mathrm{ng} / \mathrm{dl}$ and those with insufficiency (or profound deficiency) where the level in $<10$ $\mathrm{ng} / \mathrm{dl}$ while the normal level in $20-50 \mathrm{ng} / \mathrm{dl}$.

Only 2 patients of SADIS group and 1 patient of MGB group suffered insufficiency which could explain the hypocalcaemia of $13.3 \%$ in SADIS and $6.6 \%$ in MGB group. Poor compliance was reported in these patients regarding vitamin supplementation in general. That's why higher doses of vitamins and patient education was implemented and further follow up at close time interval was advised.

A study done by Sánchez-Pernaute et al. (17) showed Vitamin B12 deficiency reported in $8 \%$ of SADIS patients after one year. Another study done by Sánchez-Pernaute et al. (9) on patients one year after SADIS showed $16 \%$ of patients with anaemia and $20 \%$ of patients with low level of Vit B12.

A study done by Wang et al. ${ }^{(24)}$ showed incidence of anemia in $9.7 \%$ in MGB patients whereas another study by Rutledge and Walsh (14) showed incidence of anaemia of $4.9 \%$ after MGB.

Reporting the anaemia incidence appears to be more general rather than specific description and sometimes misleading term without specification of being macrocytic anaemia which can be attributed to Vit B12 or folate deficiency or microcytic anaemia which can be due to iron deficiency which may be attributed to poor absorption after surgery.

Two of the three anaemic patients of SADIS group had menorrhagia under investigation and one of the two anaemic MGB patients had chronic epistaxis which could be the cause or the exacerbating factor for their iron deficiency anaemia beside defective iron and Vit B12 absorption after surgery.

However, the postoperative 12 months mean level of albumin is $3.75 \pm 0.12 \mathrm{gm} / \mathrm{dl}$ in SADIS group vs $3.81 \pm 0.24 \mathrm{gm} / \mathrm{dl}$ in MGB group which appears to be at the low normal level (normal level 3.5-5.5 gm/dl) with slightly more albumin-lowering effect of SADIS operation than MGB operation.

The main limitation of the present study is the number of patients which is relatively low, and this could affect long-term results, as the missing of one or 2 patients can induce great variations in the results. Accordingly, future studies including more patients in both groups are highly advised to minimize statistical errors.

Hereby, we also recommend extended follow up of large number of patients throughout longer postoperative periods to detect any weight regain, relapse of metabolic diseases or progression of nutritional deficiencies.

Moreover, a comparison to duodenal switch is also necessary to compare the effectiveness in weight loss and diabetic remission, and the reduction in postoperative and long-term complications especially nutritional outcome to obtain a definitive answer about the presumed benefit of the elimination of one anastomosis as compared to classic duodenal switch.

Meticulous study of iron profile to accurately correlate to the anaemia type would be advised in further studies as well as measuring PTH levels with Vitamin D level as part of calcium homeostasis assessment to exclude any other pathological conditions and specifically attribute the deficiency to the actual cause whether defective absorption or other causes. 


\section{Alaa Abbas Sabry Moustafa, et al.,}

\section{Conclusion}

When compared to gastric bypass, SADI-S appears to be an effective and safe therapeutic technique as a revisional surgery for failed primary SG with excellent shortterm results for treating morbid obesity and its associated comorbidities with a low rate of nutritional complications. Proving its safety and efficacy by further studies and long term follow up will grant it more popularity in the future.

\section{REFERENCES:}

1. Fontaine KR, Redden DT, Wang C, et al. (2003): Years of life lost due to obesity. Jama. 289(2):187-93.

2. Sjostrom L, Narbro K, Sjostrom CD, et al. (2007): Effects of bariatric surgery on mortality in Swedish obese subjects. N Engl J Med; 357:741—52.

3. Fuks D, Verhaeghe $\mathrm{P}$, Brehant $\mathrm{O}$, et al. (2009): Results of laparoscopic sleeve gastrectomy: a prospective study in 135 patients with morbid obesity. Surgery; 145:106-13.

4. Weiner RA, Theodoridou $\mathrm{S}$, Weiner $\mathrm{S}$ (2011): Failure of laparoscopic sleeve gastrectomy--further procedure? Obes Facts 4 Suppl 1: 42-6.

5. Himpens J, Dobbeleir J, Peeters G, et al. (2010): Long-term results of laparoscopic sleeve gastrectomy for obesity. Ann Surg; 252:319-24.

6. Rebibo L, Fuks D, Verhaeghe P, et al. (2012): Repeat sleeve gastrectomy compared with primary sleeve gastrectomy: a single-center, matched case study. Obes Surg; 22:1909-12
7. Gumbs AA, Pomp A, Gagner $\mathrm{M}$, et al. (2007): Revisional bariatric surgery for inadequate weight loss. Obes Surg;17:1137-45.

8. Lee WJ, Yu PJ, and Wang W, (2005): Laparoscopic Roux-en-Y versus minigastric bypass for the treatment of morbid obesity: a prospective randomized controlled clinical trial. Ann Surg; 242:208.

9. Sánchez-Pernaute A and Torres AJ (2013): Sleeve to Duodenal Switch/SADI-S, Bariatric Times; 10(5 Suppl A): A20-1.

10. Sánchez-Pernaute A (2010): Laparoscopic bariatric surgery. Surg Endosc 20 Suppl: S450.

11. Scopinaro N, Adami GF, Marinari GM, et al. (1998): Biliopancreatic diversion. World J Surg. 22:936-46.

12. Rutledge $\mathrm{R}$ (2001): The mini-gastric bypass: experience with the first 1272 cases. Obes Surg; 11:276- 280.

13. Carbajo M, García-Caballero M, Toledano M (2005): One- anastomosisgastric bypass by laparoscopy; results of the first 209 patients. ObesSurg; 15(3):398-404.

14. Rutledge $\mathrm{R}$ and Walsh TR (2005): "Continued excellent results with the minigastric bypass: six-year study in 2,410 patients". Obes Surg.; 15(9):1304-8.

15. Noun R, Skaff J, Riachi E, et al. (2012): One thousand consecutive minigastric bypass: short- and long- term outcome. Obes Surg; 22(5): 697-703.

16. Sánchez-Pernaute A, Miguel Angel R, Pablo T, et al. (2011): SADI-S: Weight loss and metabolic results in the first 3 years: Surgery for Obesity and Related Diseases 7; 372-416. 
17. Sánchez-Pernaute A, Rubio M, Cabrerizo L, et al. (2015): Single-anastomosis duodenoileal bypass with sleeve gastrectomy (SADI-S) for obese diabetic patients. Surgery for Obesity and Related Diseases; 11:1092-8.

18. Del Gid EA, Sanchez P, Esther SL, et al. (2014): Single anastomosis duodeno-ileal bypass with sleeve gastrectomy: mid-term results of a novel technique compared with laparoscopic gastric bypass: j. am. Coll. surg. 07.392

19. Schauerre PR, Burguera B, Ikramuddin S, et al. (2003) Effect of laparoscopic Roux-en $\mathrm{Y}$ gastric bypass on type 2 diabetes mellitus. Ann Surg; 238(4):467-85.

20. Ramos-Leví AM, Rubio C, Herrera MA (2014): Metabolic surgery: quovadis ? Endocrinol Nutr; 61(1):35-46.

21. Kilpatrick ES (2000): Glycated haemoglobin in the year 2000. J Clin Pathol; 53(5):335-9.
22. Ramos-Levi A, Cabrerizo L, Matía P, et al. (2013): Which criteria should be used to define type2 diabetes resmission after bariatric surgery? BMC Surgery; 13:8-10.

23. Buse JB, Caprio S, Cefalu WT, et al. (2009): How do we define cure for diabetes? Diabetes Care; 32(11):2133-5.

24. Wang W, Wei PL, Lee YC, et al. (2005): Short-term results of laparoscopic minigastric bypass. Obes Surg.; 15(5):648-54.

25. Slater GH, Ren CJ, Siegel N, et al. (2004): Serum fat- soluble vitamin deficiency an abnormal calcium metabolism after malabsorptive bariatric surgery. J Gastrointest Surg; 8:48 -55.

26. Brolin RE, LaMarca LB, Kenler HA, et al. (2002): Malabsorptive gastric bypass in patients with superobesity. J Gastrointest Surg; 6:195-203. 
مقارنة بين اجراء عملية التحويل أحادي التوصيلة لمسار الإثني عشر و اللفائفي مع تكميم المعدة و المئو

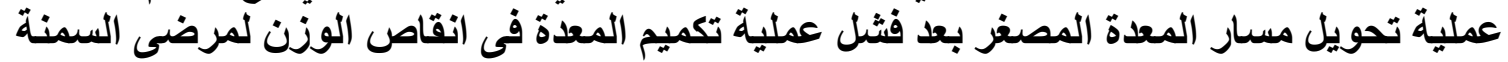
المفرطة

'علاء عباس صبرى مصطقى و'كريم صبرى عبداد محمد السميع و'عبدالله حامد ابراهيم،

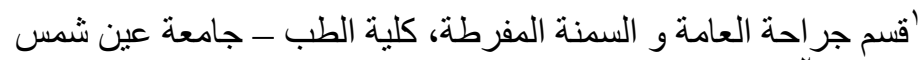

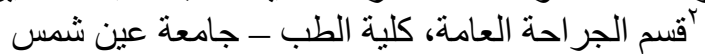

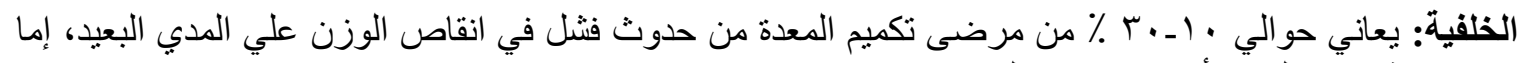
بسبب عدم كفاية فقدان الوزن أو تجدد زيادة الوزن.

الهُف: لمقارنة فقدان الوزن وكذلك الآثار الأيضية لنوعين من جر احة السمنة المفرطة بعد فثل تكميم المعدة؛ عملية

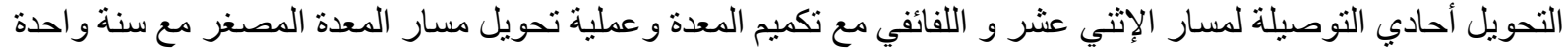
من المتابعة.

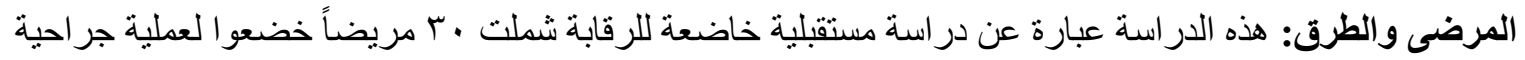

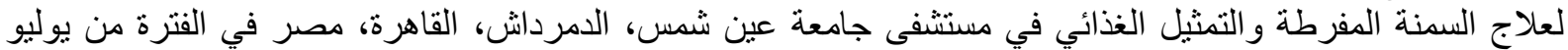

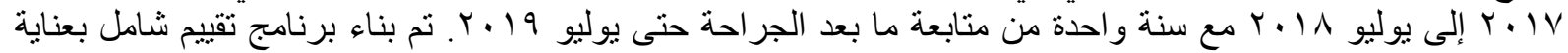

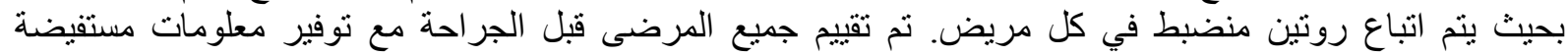
و المو افقة على المشاركة في الدر اسة.

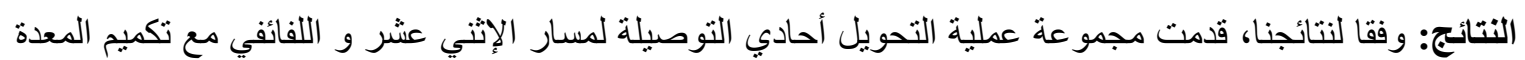

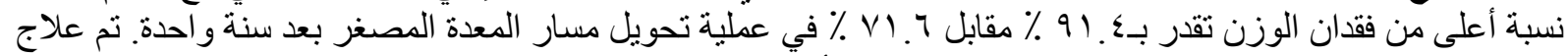

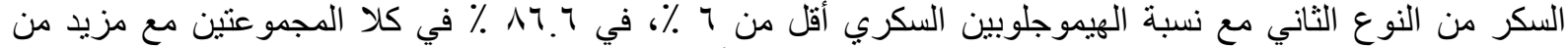

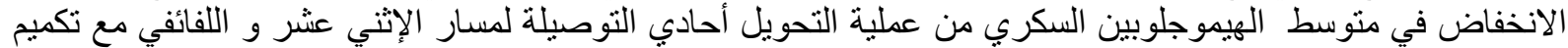

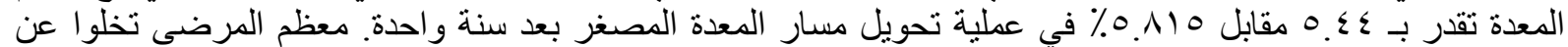

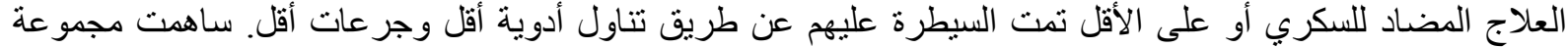

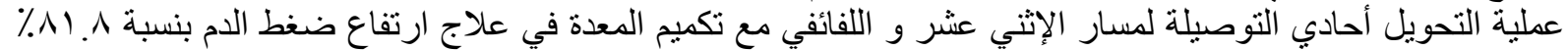

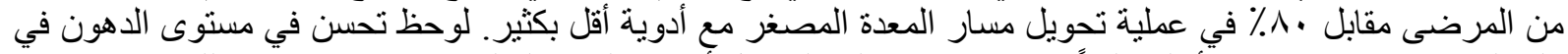

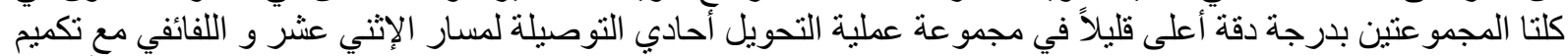

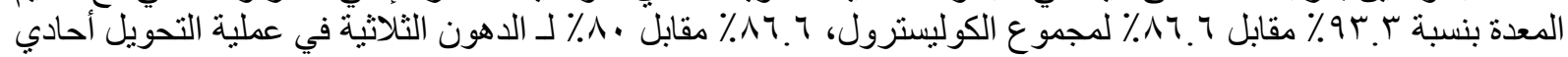

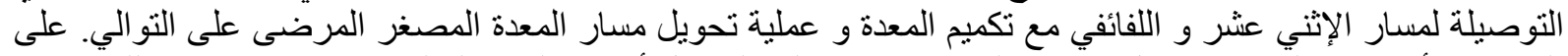

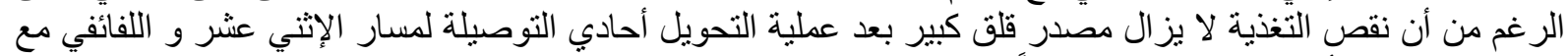

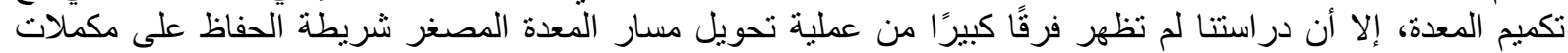

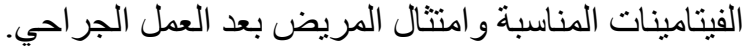

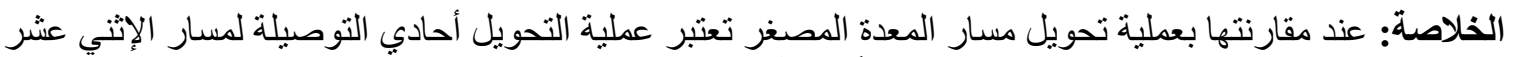

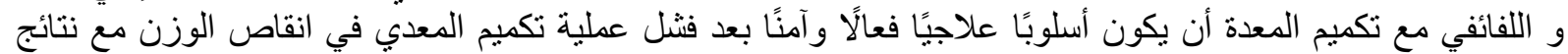

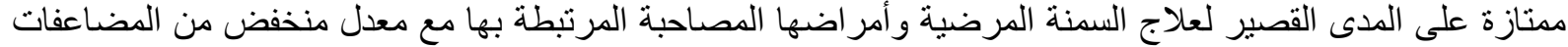

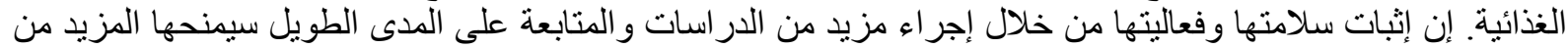
الثعبية في المستقبل.

الكلمات المفتاحية: عملية التحويل أحادي التوصيلة لمسار الإثثي عشر و اللفائفي مع تكميم المعدة، عملية تحويل 